\title{
Clinical investigation of acute myocardial infarction according to age subsets
}

\author{
JIANWEI WANG $^{1 *}$, LI LI $^{2 *}$, NING MA ${ }^{3}$, XIAOHUI ZHANG ${ }^{4}$, YANYAN QIAO ${ }^{5}$, \\ GUANGJIE FANG ${ }^{6}$, GUOPING $\mathrm{LI}^{7}$ and TAO ZHONG ${ }^{8}$ \\ ${ }^{1}$ ECG Room, Weifang Maternal and Child Health Hospital, Weifang, Shandong 261000; \\ ${ }^{2}$ ECG Room, Qianfoshan Hospital Affiliated to Shandong University, Jinan, Shandong 250014; \\ ${ }^{3}$ ECG Room, Shengli Oilfield Central Hospital, Dongying, Shandong 257034; \\ ${ }^{4}$ ECG Room, The Second Affiliated Hospital of Shandong First Medical University, Tai'an, Shandong 271000; \\ ${ }^{5}$ ECG Room, Weihai Municipal Hospital, Weihai, Shandong 234200; \\ ${ }^{6}$ ECG Room, Tai'an Traditional Chinese Medicine Hospital, Tai'an, Shandong 271000; \\ ${ }^{7}$ ECG Room, Dongying People's Hospital, Dongying, Shandong 257091; \\ ${ }^{8}$ ECG Room, Weifang People's Hospital, Weifang, Shandong 261041, P.R. China
}

Received March 23, 2020; Accepted July 17, 2020

DOI: $10.3892 /$ etm.2020.9248

\begin{abstract}
A growing number of 'Young' patients less than 40 years of age are being hospitalized with a diagnosis of acute myocardial infarction (AMI) due to increased prevalence of risk factors for atherosclerosis. The aim of this study was to compare clinical characteristics and performances of AMI between young and elderly patients. We conducted a retrospective study to compare AMI in young patients and elder patients. Based on the medical record databases in our hospital, we enrolled 114 'young' AMI patients (age $\leq 42$ years) and 179 'elder' AMI patients ( $\geq 60$ years), and then collected and analyzed their demographic information, clinical performances, and coronary angiography results. In the young AMI group, the proportion of male patients was higher than that in the elder AMI group (94.7 vs. 64.2\%, $\mathrm{P}<0.05$ ). Compared with the elder AMI patients, young patients had higher rates of smoking history and positive family medical history, but lower rates of hypertension and diabetes. Elder patients with AMI were more likely to develop various clinical performances, and multiple-branch lesions; however, young AMI patients had relatively fewer symptoms, and the tissue lesions were more limited. The clinical profiles of AMI in young patients were different from that in elder AMI patients. Specific interventions should be carried out to prevent and control the prevalence of AMI in the young population.
\end{abstract}

Correspondence to: Dr Tao Zhong, ECG Room, Weifang People's Hospital, 151 Guangwen Street, Kuiwen, Weifang, Shandong 261041, P.R. China

E-mail: zvzi46@163.com

*Contributed equally

Key words: acute myocardial infarction, young population, demography, clinical symptoms, risk factors, coronary angiography

\section{Introduction}

Acute myocardial infarction (AMI) is one of the most severe cardiac emergencies in internal medicine. Because of its properties of acute onset, quick progression, and high morbidity and mortality $(1,2)$, AMI has been a research focus for a long time. However, many recent studies have reported that the incidence of AMI has started to decrease, and this reduction is mainly concentrated in the age population greater than 60 years (3-6). Considering that elderly individuals are the main population with a high AMI incidence (7), this reduced incidence as mentioned above was a delightful change. Yet, the incidence of AMI among the young population has gradually shown a rising trend $(6,8,9)$. The young population who suffers AMI can show many clinical symptoms, such as ischemic chest pain, and the clinical course is extremely short. Some patients even experience sudden cardiac death. Although epidemiological research has determined that various behaviors and symptoms, including smoking, hypertension, diabetes, and family history of coronary heart disease (CHD), may be potential risk factors for AMI occurrence (10-12), it is still unclear how these factors differ between the young and old population. Therefore, investigating the epidemiology and clinical characteristics of AMI occurrence in the young population is a new future research focus. Through our study, the behavioral and clinical characteristics of AMI in the young population will be explored and determined, which will provide significant clues for explaining the increasing trend of AMI in the young population and helping to design specific strategies to prevent and treat young AMI patients.

\section{Patients and methods}

Study design. This retrospective, single-center, observational study was conducted during a 24-month period from January 2014 to December 2015 at Weifang People's Hospital. Consecutive patients who were diagnosed with AMI were 
included in the present study, and the criteria for enrollment in this study were based on AMI diagnostic criteria from WHO (https://doi.org/10.1016/S0140-6736(08)61237-4): At least covering two of the following three features, including ischemic thoracodynia, ECG dynamic change, and serum biomarkers of myocardial necrosis. All enrolled patients were divided in two groups, including a young group $(n=114)$ and an elder group $(\mathrm{n}=179)$. In addition, 60 young volunteers received health checkup and the same healthy subjects during January 2014 to December 2015 at Weifang People's Hospital were enrolled as the healthy control group (age range 18-72 years, with an average age of $42.1 \pm 6.3$ years). Detailed information is listed in Table I.

Data collection. In the present study, three types of information were collected. Firstly, data of AMI-related risk factors were collected, which included blood pressure, blood fat, blood glucose, smoking history, drinking history, body mass index (BMI), family history of coronary heart disease (CHD), history of hypertension and diabetes. Among these, blood fat included total cholesterol (TC), triglyceride (TG), high-density lipoprotein (HDL) and low density lipoprotein (LDL) levels. BMI was calculated using weight $(\mathrm{kg})$ and height $(\mathrm{m})$ as $\left(\mathrm{BMI}=\mathrm{kg} / \mathrm{m}^{2}\right)$, and BMI $>25$ was defined as overweight. TC $>5.75 \mathrm{mmol} / 1$ and $\mathrm{TG}>1.7 \mathrm{mmol} / 1$ were defined as being increased. Smoking history was defined as the smoking of 1 or more cigarettes per day and lasting for over 1 year; smoke 20 or more cigarettes per day and lasting for 10 years was defined as smoking heavily. Drinking was defined as drinking wine ( $>50$ g per day); drinking wine (>150 g per day) and lasting for 10 years was defined as excessive drinking. Then, we investigated whether patients had angina or not before AMI. We determined whether thoracodynia was typical or not, and whether this was combined with complications, including arrhythmia, cardiac insufficiency, formation of ventricular aneurysm, perforation of ventricular septum, cardiac shock, and death. Finally, the results of radiography were also collected. The criteria for judgment was by means of diameter measurement: Stenosis $\geq 50 \%$ was defined as a significant lesion. Lesions involved in 1 branch, 2 branches, or 3 branches of left main/anterior descending coronary artery, left circumflex, and right coronary artery was defined as 1-branch-lesion, 2-branch-lesion, and 3-branch-lesion, respectively.

Statistical analysis. In the present study, we used SAS 9.2 (SAS Institute, Inc.) to deal with data cleaning and analysis. Mean \pm SD (standard deviation) was used to reflect the distribution of measurement data, and t-test was used to compare data from two groups. $\chi^{2}$ test was used to test the differences of categorical data from two groups. $\mathrm{P}<0.05$ was defined as indicative of statistical significance, and all tests were two sided.

\section{Results}

Demographic characteristic. In the present study, we finally enrolled 293 AMI patients, including 114 young AMI patients (age $\leq 42$ years) assigned to the young group and 179 elder AMI patients (age $\geq 60$ years) assigned to the elder group. We also enrolled 60 young volunteers who received a health checkup as the healthy control group, and detail information is listed in the Table I. Among the young group, 108 (94.7\%) patients were male and $6(5.3 \%)$ patients were female. The range of age was from 32 to 44 years and the mean age was $36.6 \pm 4.0$ years. Among the elder group, $115(64.2 \%)$ patients were male and $64(35.8 \%)$ patients were female; the maximum age was 81 years and the minimum was 60 years, with an average age of $69.4 \pm 6.2$ years.

Analysis of AMI-related factors between the young and elder group. As documented in Table I, the compositions of male and female in these two groups showed a significant difference. In the young AMI group, the males had a higher rate $(94.7 \%)$ than that in the elder group $(64.2 \%)$. The percentages of males and females in the elder group were much closer. The young AMI group had a higher percentage of smoking history compared to the elder group ( 84.2 vs. $58.7 \%$; $\mathrm{P}<0.05)$ and positive family medical history (52.6 vs. 16.2\%); however, the elder AMI group seemed to be more likely to have hypertension compared with the young group (67.6 vs. 26.3\%; $\mathrm{P}<0.01)$ and diabetes (37.4 vs. $7.0 \% ; \mathrm{P}<0.01)$. For hyperlipemia and obesity, there were no obvious differences between the two groups (hyperlipemia: 45.6 vs. $54.2 \%, \mathrm{P}>0.05$; obesity: 26.3 vs. $32.4 \%, \mathrm{P}>0.05)$. Lipid metabolism in AMI patients is a key point that requires more attention. In the present study, the blood fat levels including TC and TG were significantly higher in young group compared to the elder group (TC: $8.19 \pm 2.17$ vs. $5.98 \pm 1.13, \mathrm{P}<0.05$; TG: $4.71 \pm 1.17$ vs. $2.18 \pm 0.69$, $\mathrm{P}<0.05)$. However, we did not observed the significant difference in the serum HDL and LDL levels between these two groups which may due to a relative small sample size.

Clinical performance comparisons between the young and elder group. As documented in Table II, for clinical performances, we found that young AMI patients had a higher rate of typical thoracodynia which occurred most suddenly when compared with the elder group (93.0 vs. 68.2\%; $\mathrm{P}<0.01)$. However, in the elder patients, they were more likely to have different types of clinical performances than the young patients, including premonitory symptom (84.4 vs. 33.3\% yes), arrhythmia (78.2 vs. $52.6 \%$ yes), heart failure (41.9 vs. $21.1 \%$ yes), and cardiac shock (37.4 vs. $3.5 \%$, yes).

Outcomes from coronary angiography $(C A G)$. As documented in Table III, the different age groups had different major lesion sites and statuses. The lesion per capita in the young AMI group was 1.8, which was lower than 2.8 in the elder AMI group. The lesions in the young patients mostly focused on anterior descending coronary artery, and $62.4 \%$ of lesions were one-branch lesion. Comparatively, the elder patients had more severe conditions. The distributions of lesion sites were almost equally on left main/anterior descending coronary artery, left circumflex, and right coronary artery. Three-branch lesion was the most frequent lesion, followed by two-branch lesion and one-branch lesion, which was a little different from that in the young AMI group.

Odds ratios between the young and elder group. As shown in Fig. 1, compared with the elderly group, the young males had a history of smoking and had a positive family medical history of heart disease, and had higher risk of acute myocardial 
Table I. Baseline characteristics associated with AMI between the young and elder group.

\begin{tabular}{|c|c|c|c|c|}
\hline Factors & Control group $\mathrm{n}$ & Young group n (\%) & Elder group n (\%) & P-value ${ }^{a}$ \\
\hline Total & 60 & 114 & 179 & \\
\hline Sex & & & & $<0.01$ \\
\hline Male & 31 & $108(94.7)$ & $115(64.2)$ & \\
\hline Female & 30 & $6(5.3)$ & $64(35.8)$ & \\
\hline History of smoking & & & & $<0.05$ \\
\hline Yes & 14 & $96(84.2)$ & $105(58.7)$ & \\
\hline No & 46 & $18(15.8)$ & $74(41.3)$ & \\
\hline Hypertension & & & & $<0.01$ \\
\hline Yes & 6 & $30(26.3)$ & $121(67.6)$ & \\
\hline No & 54 & $84(73.7)$ & $58(32.4)$ & \\
\hline Hyperlipemia & & & & $>0.05$ \\
\hline Yes & 3 & $52(45.6)$ & $97(54.2)$ & \\
\hline No & 57 & $62(54.4)$ & $82(45.8)$ & \\
\hline Obesity & & & & $>0.05$ \\
\hline Yes & 12 & $30(26.3)$ & $58(32.4)$ & \\
\hline No & 48 & $84(73.7)$ & $121(67.6)$ & \\
\hline Diabetes & & & & $<0.01$ \\
\hline Yes & 2 & $8(7.0)$ & $67(37.4)$ & \\
\hline No & 58 & $106(93.0)$ & $112(62.6)$ & \\
\hline Positive family medical history & & & & $<0.01$ \\
\hline Yes & 5 & $60(52.6)$ & $29(16.2)$ & \\
\hline No & 55 & $54(47.4)$ & $150(83.8)$ & \\
\hline \multicolumn{5}{|l|}{ Blood fat level (mmol/l) } \\
\hline $\mathrm{TC}$ & $3.19 \pm 0.39$ & $8.19 \pm 2.17$ & $5.98 \pm 1.13$ & $<0.05$ \\
\hline TG & $0.97 \pm 0.26$ & $4.71 \pm 1.17$ & $2.18 \pm 0.69$ & $<0.05$ \\
\hline HDL & $1.93 \pm 0.38$ & $1.66 \pm 0.42$ & $1.39 \pm 0.21$ & $>0.05$ \\
\hline LDL & $2.34 \pm 0.71$ & $3.77 \pm 0.85$ & $3.14 \pm 0.74$ & $>0.05$ \\
\hline
\end{tabular}

Young group, age $<42$ years; elder group, age $\geq 60$ years. AMI, acute myocardial infarction; TC, total cholesterol; TG, triglyceride; HDL, high-density lipoprotein; LDL, low density lipoprotein. ${ }^{\text {P }}$-values comparing the young and elder group.

infarction. However, the absence of diabetes was found to be a protective factor. Details are shown in Fig. 1.

\section{Discussion}

In this study, we analyzed clinical data collected from Weifang People's Hospital to evaluate the acute myocardial infarction (AMI) characteristics in young patients, which could provide some clues for AMI diagnosis and therapy in different age subsets in the future.

In the young AMI group (age $\leq 42$ years), the AMI incidence in males was obviously higher than that of females, which was consistent with a previous study (13). Estrogen is an important hormone protecting women from AMI (14), which involves the regulation of lipometabolism, inhibition of platelet activation, and suppression of intima proliferation caused by lesions. After menopause, the estrogen level decreases. Along with this, the protection from AMI also become weak, even disappearing, which increases the female AMI incidence (15). Smoking, hypertension, hyperlipemia, diabetes, and family

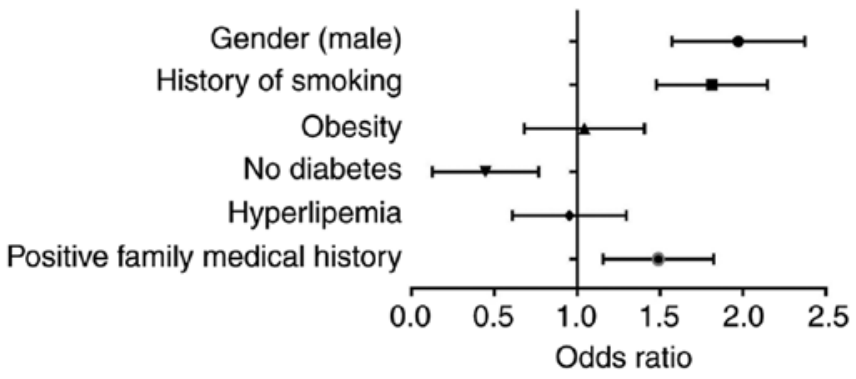

Figure 1. Adjusted odds ratios and $95 \%$ confidence intervals between the young and elder AMI group. AMI, acute myocardial infarction.

history of cardiac heart disease (CHD) are potential risk factors for the occurrence of CHD (16). The present study found that $84.2 \%$ of young patients had a history of smoking, which was higher than that of the elder group (age $\geq 60$ years). A previous study revealed that $\mathrm{CO}_{2}$ and nicotine in smoke cause hypoxia in tissues and the myocardium, which then induced coronary 
Table II. Comparisons of clinical performances between the young and elder group.

\begin{tabular}{lcc}
\hline Factors & Young group n $(\%)$ & Elder group n $(\%)$ \\
\hline Total & 114 & 179 \\
Premonitory symptom & $38(33.3)$ & $151(84.4)$ \\
Yes & $76(66.7)$ & $28(15.6)$ \\
No & & $122(68.2)$ \\
Typical thoracodynia & $106(93.0)$ & $57(31.8)$ \\
Yes & $8(7.0)$ & $140(78.2)$ \\
No & & $39(21.8)$ \\
Arrhythmia & $60(52.6)$ & \\
Yes & $54(47.4)$ & $75(41.9)$ \\
No & & $104(58.1)$ \\
Heart failure & $24(21.1)$ & $<0.01$ \\
Yes & $90(78.9)$ & $67(37.4)$ \\
No & & $112(62.6)$ \\
Cardiac shock & $4(3.5)$ & $<0.05$ \\
Yes & $110(96.5)$ & $<0.01$ \\
No & & \\
\hline
\end{tabular}

Young group, age $<42$ years; elder group, age $\geq 60$ years. AMI, acute myocardial infarction. ${ }^{\text {a }}$-values comparing the young and elder group.

Table III. Comparisons of the outcomes of coronary angiography (CAG) between the young and elder group.

\begin{tabular}{|c|c|c|c|}
\hline Factors & Young group n (\%) & Elder group n $(\%)$ & P-value ${ }^{a}$ \\
\hline Total & 114 & 179 & \\
\hline Total lesions on coronary artery & 194 & 465 & \\
\hline Lesions per capita & 1.8 & 2.6 & $<0.01$ \\
\hline Lesions on anterior descending coronary artery & & & $<0.01$ \\
\hline Yes & $101(52.1)$ & $153(32.9)$ & \\
\hline No & $93(47.9)$ & $312(67.1)$ & \\
\hline Lesions on right coronary artery & & & $>0.05$ \\
\hline Yes & $42(21.6)$ & $112(24.1)$ & \\
\hline No & $152(78.4)$ & $353(75.9)$ & \\
\hline Lesions on left circumflex & & & $>0.05$ \\
\hline Yes & $40(20.6)$ & $103(22.2)$ & \\
\hline No & $154(79.4)$ & $362(77.8)$ & \\
\hline Lesions on left main coronary artery & & & $<0.01$ \\
\hline Yes & $11(5.7)$ & $97(20.9)$ & \\
\hline No & $183(94.3)$ & $368(79.1)$ & \\
\hline \multicolumn{4}{|l|}{ Coronary angiography lesion (individual) } \\
\hline No lesions & $6(5.3)$ & $2(1.1)$ & $<0.01$ \\
\hline One-branch lesion & $71(62.4)$ & $44(24.6)$ & $<0.01$ \\
\hline Two-branch lesion & $23(20.1)$ & $56(31.3)$ & $<0.01$ \\
\hline Three-branch lesion & $14(12.2)$ & $77(43.0)$ & $<0.01$ \\
\hline
\end{tabular}

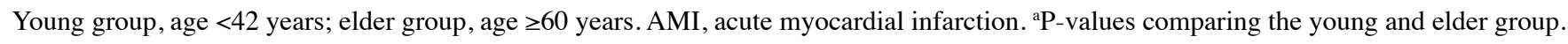

spasm and an increase in blood viscosity (17). A history of long-term smoking decreases coronary dilation function, and increases platelet aggregation (18). Meanwhile, high-density lipoprotein is decreased and low-density lipoprotein is 
increased. This change damages serum antioxidative function (19). Based on the above clinical situations, coronary atherosclerotic plaque forms, and is aggravated, which in turn promotes the occurrence and progression of CHD. Thus, abstinence from smoking is one of the most critical interventions for CHD prevention, especially for young people

The rate of hypertension and diabetes in the elder group were higher than that in the young group, which suggests that the elderly AMI patients were consistently accompanied by certain chronic diseases (20). At the same time, the elderly people had a higher frequency of complications, including arrhythmia, heart failure, and cardiac shock, which were the significant reasons for the poor prognosis in AMI elder patients. Noticeably, the rate of hypertension and various complications in young patients were not low. In the present study, the incidence of hypertension and hyperlipemia in the young AMI patients were 26.3 and $45.6 \%$, which were significantly higher than these percentages in the young health control group, and should be focused on. The rate of hyperlipemia did not show much difference between the young and elder groups, but almost half of the patients had hyperlipemia in the two groups. Some studies have shown that high levels of serum total cholesterol (TC) and low density lipoprotein (LDL) are independent risk factors for coronary disease (21-23). Therefore, controlling blood fat is an important intervention for reducing AMI incidence in all populations.

Through the coronary angiography (CAG) results, we found that the most frequent coronary lesion in young AMI patients was the one-branch lesion (62.4\%), and the secondary injury was limited. Comparatively, elder AMI patients had more multiple-branch lesions and calcified lesions, which has serious influences on cardiac function. Additionally, healthy coronary artery with only none or mild atherosclerosis, normal coronary angiography and negative treadmill test was found in a certain proportion of young AMI patients, which are consistent with previous reports $(24,25)$. This may be related to the clinical characteristics of the young AMI patients, who exhibited a short course of disease and fewer complications of hypertension and diabetes.

After comparing the risk factors and clinical performances between young AMI patients and elder AMI patients comprehensively, we found that smoking, hyperlipemia, and positive family medical history were the most critical factors for young AMI patients, which may play important roles in increasing AMI incidence. Interventions for controlling these risk factors will be important in the future for young patients, including abstinence from smoking, alcohol consuming limitation, proper exercise and a rational diet.

\section{Acknowledgements}

Not applicable.

\section{Funding}

No funding was received.

\section{Availability of data and materials}

The datasets used and/or analyzed during the current study are available from the corresponding author on reasonable request.

\section{Authors' contributions}

JW and LL designed the study and drafted the manuscript. NM, XZ and YQ were responsible for the collection and analysis of the experimental data. GF, GL and TZ analyzed AMI-related factors and compared clinical performance. All authors read and approved the manuscript and agree to be accountable for all aspects of the research in ensuring that the accuracy or integrity of any part of the work are appropriately investigated and resolved.

\section{Ethics approval and consent to participate}

The study was approved by the Ethics Committee of W.F. Maternal and Child Health Hospital, Weifang, Shandong, P.R. China. Patients who participated in this research, signed the informed consent and had complete clinical data. Signed written informed consents were obtained from the patients and/or guardians.

\section{Patient consent for publication}

Not applicable.

\section{Competing interests}

The authors declare that they have no competing interests.

\section{References}

1. Anderson JL and Morrow DA: Acute myocardial infarction. N Engl J Med 376: 2053-2064, 2017

2. Longjian L, Ling A and Xinglong Li: Analysis of death cause in the total population in Mentougou district of Beijing from 2006 to 2015. Xian Dai Yu Fang Yi Xue 44: 1823-1826, 2017.

3. Randall SM,Zilkens R, Duke JM and Boyd JH: Western Australia population trends in the incidence of acute myocardial infarction between 1993 and 2012. Int J Cardiol 222: 678-682, 2016.

4. Schmidt M, Jacobsen JB, Lash TL, Bøtker HE and Sørensen HT: 25 year trends in first time hospitalisation for acute myocardial infarction, subsequent short and long term mortality, and the prognostic impact of sex and comorbidity: A Danish nationwide cohort study. BMJ 344: e356, 2012.

5. Dégano IR, Salomaa V, Veronesi G, Ferriéres J, Kirchberger I, Laks T, Havulinna AS, Ruidavets JB, Ferrario MM, Meisinger C, et al: Twenty-five-year trends in myocardial infarction attack and mortality rates, and case-fatality, in six European populations. Heart 101: 1413-1421, 2015.

6. Wang DZ, Shen CF, Zhang Y, Zhang H, Song GD, Li W, Xue XD, Xu ZL, Zhang S and Jiang GH: Fifteen-year trend in incidence of acute myocardial infarction in Tianjin of China. Zhonghua Xin Xue Guan Bing Za Zhi 45: 154-159, 2017 (In Chinese).

7. Hernandez-Suarez DF, Osterman-Pla AD, Carrasquillo O, Aranda J,Baez S,Lopez Mand Garcia-Rivera EJ:Epidemiological profile of hispanics admitted with acute myocardial infarction in Puerto Rico: The Experience of 2007, 2009 and 2011. J Clin Med Res 9: 528-533, 2017.

8. Guo X, Li Z, Vittinghoff E, Sun Y and Pletcher MJ: Trends in rate of acute myocardial infarction among patients aged $<30$ years. Nat Rev Cardiol 15: 119, 2018.

9. Liu Y, Han T, Gao M, Wang J, Liu F, Zhou S and Chen Y: Clinical characteristics and prognosis of acute myocardial infarction in young smokers and non-smokers ( $\leq 45$ years): A systematic review and meta-analysis. Oncotarget 46: 81195-81203, 2017.

10. Veeranna V, Zalawadiya SK, Niraj A, Pradhan J, Ference B, Burack RC, Jacob S and Afonso L: Homocysteine and reclassification of cardiovascular disease risk. J Am Coll Cardiol 58: 1025-1033, 2011. 
11. Sailam V, Karalis DG, Agarwal A, Alani F, Galardi S, Covalesky V and Athanassious C: Prevalence of emerging cardiovascular risk factors in younger individuals with a family history of premature coronary heart disease and low Framingham risk score. Clin Cardiol 31: 542-545, 2008.

12. Ueshima H, Sekikawa A, Miura K, Turin TC, Takashima N, Kita Y, Watanabe M, Kadota A, Okuda N, Kadowaki T, et al: Cardiovascular disease and risk factors in Asia: A selected review. Circulation 118: 2702-2709, 2008.

13. Matsis K, Holley A, Al-Sinan A, Matsis P, Larsen PD and Harding SA: Differing clinical characteristics between young and older patients presenting with myocardial infarction. Heart Lung Circ 26: 566-571, 2017.

14. Manson JE, Allison MA, Rossouw JE, Carr JJ, Langer RD, Hsia J, Kuller LH, Cochrane BB, Hunt JR, Pettinger MB, et al Estrogen therapy and coronary-artery calcification. N Engl J Med 356: 2591-2602, 2007.

15. Sidney S, Petitti DB and Quesenberry CP Jr: Myocardial infarction and the use of estrogen and estrogen-progestogen in postmenopausal women. Ann Intern Med 127: 501-508, 1997.

16. Ebrahim S, Taylor F, Ward K, Beswick A, Burke M and Davey Smith G: Multiple risk factor interventions for primary prevention of coronary heart disease. Cochrane Database Syst Rev: Jan 19, 2011 (Epub ahead of print). doi: 10.1002/14651858. CD001561.pub3.

17. Lakier JB: Smoking and cardiovascular disease. Am J Med 93 (Suppl 1): S8-S12, 1992.

18. Cavusoglu Y, Timuralp B, Us T, Akgün Y, Kudaiberdieva G, Gorenek B, Unalir A, Goktekin O and Ata N: Cigarette smoking increases plasma concentrations of vascular cell adhesion molecule-1 in patients with coronary artery disease. Angiology 55: 397-402, 2004
19. Yan S, Xiaopeng Z, Xudong J, Wenzhong Z, Wei W, Wenming C, Xin $Z$ and Ning L: Potential mechanisms of oxidative damage mechanisms by smoking. Teratog Carcinog Mutagen 21: 50-53, 2009.

20. Maosen W, Shengfeng W and Zitian Z: Clinical characteristics and treatment of old acute myocardial infarction patients. Contemp Med 17: 56-57, 2011.

21. The Lipid Research Clinics Coronary Primary Prevention Trial results. II. The relationship of reduction in incidence of coronary heart disease to cholesterol lowering. JAMA 251: 365-374, 1984.

22. Sever PS, Dahlöf B, Poulter NR, Wedel H, Beevers G, Caulfield M, Collins R, Kjeldsen SE, Kristinsson A, McInnes GT, et al: Prevention of coronary and stroke events with atorvastatin in hypertensive patients who have average or lower-than-average cholesterol concentrations, in the Anglo-Scandinavian Cardiac Outcomes Trial--Lipid Lowering Arm (ASCOT-LLA): A multicentre randomised controlled trial. Lancet 361: 1149-1158, 2003.

23. Baigent C, Keech A, Kearney PM, Blackwell L, Buck G, Pollicino C, Kirby A, Sourjina T, Peto R, Collins R, et al: Efficacy and safety of cholesterol-lowering treatment: Prospective meta-analysis of data from 90,056 participants in 14 randomised trials of statins. Lancet 366: 1267-1278, 2005.

24. Wenlin R, Dayi H, Jianjun P, et al: Clinical analysis of percutaneous coronary intervention in young AMI patients. Beijing Med J 26: 423-424, 2004.

25. Xuegang Y and Chuanrong C: Analysis of clinical characteristics and CAG results between old and young AMI patients. J Clin Cardiol 13: 281-365, 1997. 